\title{
STUDIES OF UREA EXCRETION. IX. COMPARISON OF UREA CLEARANCES CALCULATED FROM THE EXCRETION OF UREA, OF UREA PLUS AMMONIA, AND OF NITROGEN DETERMINABLE BY HYPOBROMITE
}

\author{
By DONALD D. VAN SLYKE, IRVINE H. PAGE, ALMA HILLER AND ESBEN KIRK \\ (From the Hospital of the Rockefeller Institute for Medical Research, New York)
}

(Received for publication July 26, 1935)

According to the usually accepted concept, the urea removed from the blood by the kidneys is excreted in two forms, chiefly as urea itself, but partly as ammonia. That the ammonia of the urine is formed in the kidneys was demonstrated by Nash and Benedict (1921), in work which has been confirmed by other authors (cf. Peters and Van Slyke, I, p. 373). Evidence from animal experiments in vivo has indicated urea as the probable chief source of the ammonia formed by the kidney (cf. Peters and Van Slyke (1931) p. 373). Krebs' recent work (1933) with kidney tissue in vitro has indicated the possibility that blood amino acids may also be a direct source of the ammonia formed by the kidney, but whether an important part of the ammonia actually excreted comes from them has not been demonstrated.

If urinary ammonia is entirely or chiefly formed from the blood urea, the urea cleared from the blood per minute by the kidneys is represented more accurately by the excretion of urea + ammonia rather than of urea alone. It appears possible, therefore, that urea clearances calculated from the excretion of urea + ammonia may measure the work of the kidneys in excreting urea from the blood more accurately than clearances calculated in the usual manner from the output of urea alone.

It therefore appears to be a problem of physiological interest to ascertain whether, when the ammonia: urea ratio in urine varies markedly, more consistent values for the urea clearance are calculated from the excretion rate of urea alone, or of urea + ammonia.

For clinical interpretation, as already pointed out by Bell, Gilmour and Cameron (1934), it would seldom make a serious difference if, in the formula for calculating the urea clearance (Möller, McIntosh and Van Slyke $(1928, a))$, urine urea nitrogen were replaced by urea + ammonia nitrogen. Since the ammonia nitrogen ordinarily equals 1 to 10 per cent of the urea nitrogen, the clearance calculated from urea + ammonia would be from 1 to 10 per cent higher than that calculated from the urea excretion alone. In nephritis the proportion of nitrogen excreted as ammonia is likely to be less than in normal subjects, because the damaged kidney turns a smaller proportion than normal of the urinary nitrogen into ammonia (see literature quoted by Peters and Van Slyke (1931), p. 378; also data in Table III, present paper).

Conditions do occur, however, in which the ammonia: urea ratio may be unusually high. Such are the acidoses of diabetes and starvation, and the depression of urea output caused by very low protein, high calory, diets. If the urea clearance is measured in such a condition, it is of interest to know whether more consistent values may be expected when the calculation is based on the output of urea, or of urea + ammonia.

Aside from this, and the physiological interest of the problem, the question has some practical bearing on the convenience of procedures for determining the urea clearance. If a method for determining urinary urea is used which depends upon estimation of the ammonia formed when the urea is hydrolyzed by heat or urease, it is simpler to determine urea + ammonia than urea alone, since thereby one avoids an extra operation to remove or determine the preformed ammonia. Likewise, if the hypobromite gasometric method is used, it is simpler to determine urea + ammonia, since the reagent gives practically the same yields of $\mathrm{N}_{2}$ from both. Furthermore, if, by bladder infection or delay in analyzing urine, part of the urea is decomposed into ammonia before analysis, no error is introduced into the figure representing the sum of urea + ammonia. 
We have sought to solve the proposed problem by ascertaining whether, when the ammonia : urea ratio in the urine is greatly increased, calculation of urea clearances with, or without, inclusion of the excreted ammonia gives values more consistent with those yielded by the same subjects under ordinary conditions. In order to obtain maximal increase in the ammonia: urea ratio, the numerator was increased by inducing acidosis with ammonium or calcium chloride administered by mouth, while the denominator was decreased by putting the subjects on low protein diet. By these means the ratio could be raised from its average usual value of about 0.05 to as high as 1.0. Unless such means are taken to increase the ratio, the effect of adding the ammonia to the urea for calculation of the clearance is likely to be small (averaging about 5 per cent) in comparison with normal spontaneous variation in the clearance, which may be \pm 20 per cent, so that it would be difficult to ascertain, except by statistical data, whether the inclusion of the ammonia increased or decreased the constancy of the clearance.

We have also determined the clearances of urea with and without inclusion of the urinary ammonia in normal subjects and nephritics on ordinary diets, in order to obtain data, in addition to those of Bell, Gilmour and Cameron (1934), indicating the usual range of the effect of including the ammonia.

Finally, led by the extreme rapidity and simplicity of the approximate gasometric determination of urea + ammonia with hypobromite, we have, in a series of nephritic subjects with renal function varying from normal to that of terminal nephritis, compared urea clearances with " hypobromite nitrogen" clearances. These were calculated by substituting, in place of the urine urea nitrogen in the clearance formula, the urinary nitrogen determined by the hypobromite method, which is approximately $0.95 \times$ (urea nitrogen + ammonia nitrogen).

\section{METHODS}

Blood urea was determined by the gasometric urease method (Van Slyke, 1927). Urea in urine was determined by the gasometric urease method (Van Slyke, 1927). Ammonia in urine was determined by the aeration technique of Van Slyke and Cullen (1914, 1916).
Creatinine in blood and urine was determined by the methods of Folin (Peters and Van Slyke, 1932, pp. 602 to 604).

For the approximate hypobromite determination of ammonia + urea in urine, the manometric procedure of Van Slyke (1929) was used, with the modification that the removal of ammonia by treatment with permutit was omitted. The urine was simply diluted 10 - or 20 -fold, according to its concentration, and a sample was transferred to the manometric apparatus for the analysis. The improved hypobromite reagent of Van Slyke and Kugel (1933) was used. Twelve to 15 determinations could be done in an hour, and the procedure is so simple that the loss of an analysis, or failure of duplicates to check within 0.5 per cent, was a rarity.

\section{Calculations}

Urea clearance. The urea clearance was calculated by Formula 1 or 2 , depending on the urine volume, as described by Möller, McIntosh and Van Slyke (1928, a), (see also Peters and Van Slyke (1932), pp. 564 to 570).

$$
\begin{aligned}
& \text { (1) } C_{U r}=\frac{1.33 U V}{B} \text {, when } V>2 . \\
& \text { (2) } C_{U r}=\frac{1.85 U \sqrt{ } V}{B} \text {, when } V<2 .
\end{aligned}
$$

$C_{U r}=$ urea clearance, in per cent of average normal; $U=$ urine urea nitrogen, in mgm. per $100 \mathrm{cc}$; $B=$ blood urea nitrogen, in mgm. per $100 \mathrm{cc}$; $V=$ urine volume in cc. per minute. $V$ was corrected for body size, as described by McIntosh, Möller and Van Slyke (1928). (See also Peters and Van Slyke (1932), p. 569.)

Clearance of urea + ammonia, $\mathrm{C}_{\mathrm{Ur}+\mathrm{NH}_{\mathrm{z}}}$. The clearance of urea + ammonia, also in percentage of average normal urea clearance, was calculated by Formulae 1 and 2 , but with $U$ representing urea $+\mathrm{NH}_{3}$ nitrogen, instead of only urea nitrogen, in the urine.

Hypobromite nitrogen clearance, $C_{H y}$. This clearance was calculated by the same formulae, but with the urinary nitrogen determined by the hypobromite method substituted for $U$. In this case $U$ represents approximately 95 per cent of the urea + ammonia nitrogen.

Creatinine clearance was determined after giving 5 grams of creatinine, the calculation being like that of urea clearance by Formula 1 , with 0.68 replacing the factor 1.33 .

\section{RESULTS}

Observations in which the ammonia: urea ratio in the urine was increased by induced acidosis and low protein diet. It is obvious from Table I, dates April 23 to April 26, and Table II, dates June 7 to June 11, that when an important part of the urea + ammonia nitrogen was in the form of ammonia, the clearances calculated from the excreted urea alone were much below the usual urea clearance shown by the same subject. When the clearances were calculated from the excretion 
TABLE I

Comparison of clearances of urea and urea + ammonia in subjects before and during induced acidosis. Subjects with normal renal function

\begin{tabular}{|c|c|c|c|c|c|c|c|c|c|c|c|}
\hline Date & $\begin{array}{l}\text { Protein } \\
\text { in diet }\end{array}$ & $\begin{array}{l}\text { Acidifying } \\
\text { salt taken }\end{array}$ & $\begin{array}{c}\text { Urine } \\
\text { volume } \\
\text { corrected } \\
\text { for } \\
\text { body } \\
\text { size }\end{array}$ & $\begin{array}{c}\text { Urine } \\
\text { urea } \\
\text { nitrogen. } \\
\text { Urease } \\
\text { method }\end{array}$ & $\begin{array}{c}\text { Urine } \\
\text { ammonia } \\
\text { nitrogen } \\
\text { Van Slyke- } \\
\text { Cullen } \\
\text { aeration }\end{array}$ & $\begin{array}{c}\text { Blood } \\
\text { urea } \\
\text { nitrogen. } \\
\text { Urease } \\
\text { on whole } \\
\text { blood }\end{array}$ & $\begin{array}{l}\text { Urea } \\
\text { clear- } \\
\text { ance } \\
C_{U r}\end{array}$ & $\begin{array}{c}\text { Urea }+\mathrm{NH}_{3} \\
\text { clearance } \\
\mathrm{C}_{U r}+\mathrm{NH}_{3}\end{array} \mid$ & $\begin{array}{c}\text { Ratio of } \\
C_{U r+N H_{z}} \\
\text { to } \\
C_{U r}\end{array}$ & $\begin{array}{c}\text { Excretion } \\
\text { rate of } \\
\text { urea }+\mathrm{NH}_{3} \\
\text { calculated } \\
\text { as } \\
\text { nitrogen }\end{array}$ & $\begin{array}{c}\text { Ve- } \\
\text { nous } \\
\text { plas- } \\
\text { ma } \\
\mathrm{CO}_{2}\end{array}$ \\
\hline February 26 & $\begin{array}{c}\text { grams } \\
\text { Unlimited }\end{array}$ & $\begin{array}{c}\text { grams } \\
0\end{array}$ & $\begin{array}{c}c c . \\
\begin{array}{c}c c . \\
\text { per } \\
\text { minute }\end{array} \\
4.6 \\
9.3\end{array}$ & $\begin{array}{c}\text { mgm. } \\
\text { per } \\
100 \text { cc. } \\
185 \\
92\end{array}$ & $\begin{array}{l}\text { mgm. } \\
\text { per } \\
100 \text { cc. }\end{array}$ & $\begin{array}{c}\text { mgm. } \\
\text { per } \\
100 \text { cc. } \\
10.2 \\
10.2\end{array}$ & $\begin{array}{c}\text { per cent } \\
\text { of } \\
\text { normal } \\
112 \\
112\end{array}$ & $\left|\begin{array}{c}\text { per cent } \\
\text { of } \\
\text { normal } C_{U V}\end{array}\right|$ & & $\begin{array}{c}\text { grams } \\
\text { per } \\
24 \text { hours }\end{array}$ & $\begin{array}{c}m M . \\
\text { per } \\
\text { iter }\end{array}$ \\
\hline March 17 & Unlimited & 0 & $\begin{array}{l}5.4 \\
2.7\end{array}$ & $\begin{array}{l}166 \\
280\end{array}$ & & $\begin{array}{l}12.8 \\
12.8\end{array}$ & $\begin{array}{l}93 \\
80\end{array}$ & & & & \\
\hline March 19 & Unlimited & 0 & $\begin{array}{l}1.9 \\
1.9\end{array}$ & $\begin{array}{l}435 \\
401\end{array}$ & $\begin{array}{l}14.4 \\
10.2\end{array}$ & $\begin{array}{l}12.8 \\
12.8\end{array}$ & $\begin{array}{l}87 \\
81\end{array}$ & $\begin{array}{l}90 \\
83\end{array}$ & $\begin{array}{l}103 \\
102\end{array}$ & $\begin{array}{l}12.2 \\
11.2\end{array}$ & \\
\hline March 29 & Unlimited & $\mathrm{NH}_{4} \mathrm{Cl} \dagger$ & $\begin{array}{l}2.8 \\
4.5\end{array}$ & $\begin{array}{l}251 \\
177\end{array}$ & $\begin{array}{l}45.7 \\
30.4\end{array}$ & $\begin{array}{l}12.8 \\
12.8\end{array}$ & $\begin{array}{l}74 \\
85\end{array}$ & $\begin{array}{r}88 \\
100\end{array}$ & $\begin{array}{l}118 \\
117\end{array}$ & $\begin{array}{l}12.0 \\
13.4\end{array}$ & 22.1 \\
\hline March 30 & Unlimited & $\mathrm{NH}_{4} \mathrm{Cl}$ & $\begin{array}{l}3.5 \\
5.1\end{array}$ & $\begin{array}{l}235 \\
150\end{array}$ & $\begin{array}{l}51.6 \\
31.2\end{array}$ & $\begin{array}{l}12.9 \\
12.9\end{array}$ & $\begin{array}{l}84 \\
80\end{array}$ & $\begin{array}{r}103 \\
96\end{array}$ & $\begin{array}{l}122 \\
120\end{array}$ & $\begin{array}{l}14.4 \\
13.2\end{array}$ & 20.0 \\
\hline April 2 & Unlimited & $\mathrm{NH}_{4} \mathrm{Cl}$ & $\begin{array}{l}1.8 \\
1.7\end{array}$ & $\begin{array}{l}329 \\
354\end{array}$ & $\begin{array}{r}96.7 \\
104.5\end{array}$ & $\begin{array}{l}12.2 \\
12.2\end{array}$ & $\begin{array}{l}68 \\
70\end{array}$ & $\begin{array}{l}88 \\
91\end{array}$ & $\begin{array}{l}129 \\
129\end{array}$ & $\begin{array}{l}11.1 \\
11.4\end{array}$ & 15.4 \\
\hline April 16 & $20^{*}$ & 0 & $\begin{array}{l}3.4 \\
2.8\end{array}$ & $\begin{array}{l}104 \\
114\end{array}$ & $\begin{array}{l}7.3 \\
7.5\end{array}$ & $\begin{array}{l}6.6 \\
6.6\end{array}$ & $\begin{array}{l}70 \\
65\end{array}$ & $\begin{array}{l}75 \\
69\end{array}$ & $\begin{array}{l}107 \\
107\end{array}$ & $\begin{array}{l}5.4 \\
4.9\end{array}$ & \\
\hline April 23 & 20 & $\mathrm{CaCl}_{2} \ddagger$ & $\begin{array}{l}1.4 \\
1.0\end{array}$ & $\begin{array}{l}123 \\
148\end{array}$ & $\begin{array}{l}34.8 \\
52.4\end{array}$ & $\begin{array}{l}5.1 \\
5.1\end{array}$ & $\begin{array}{l}53 \\
55\end{array}$ & $\begin{array}{l}69 \\
74\end{array}$ & $\begin{array}{l}128 \\
135\end{array}$ & $\begin{array}{l}3.2 \\
3.0\end{array}$ & 24.2 \\
\hline April 25 & 20 & $\mathrm{CaCl}_{2}$ & 4.2 & 34.5 & 27.0 & 3.8 & 50 & 90 & 180 & 3.7 & \\
\hline April 26 & 20 & $\mathrm{CaCl}_{2}$ & $\begin{array}{l}3.8 \\
2.5\end{array}$ & $\begin{array}{l}42.8 \\
53.1\end{array}$ & $\begin{array}{l}35.9 \\
54.0\end{array}$ & $\begin{array}{l}4.3 \\
4.3\end{array}$ & $\begin{array}{l}50 \\
42\end{array}$ & $\begin{array}{l}93 \\
85\end{array}$ & $\begin{array}{l}186 \\
202\end{array}$ & $\begin{array}{l}4.1 \\
2.8\end{array}$ & 18.3 \\
\hline
\end{tabular}

* Diet with only 20 grams protein, April 15 to 25 , inclusive. Total calories 2300.

$\dagger$ Administration of 10 grams $\mathrm{NH}_{4} \mathrm{Cl}$ daily, March 27 to April 12, inclusive (=187 m.eq.).

$\ddagger$ Administration of 10 grams CaCl $2 \cdot 2 \mathrm{H}_{2} \mathrm{O}$ daily, April 21 to 26 , inclusive, equivalent to 7.55 grams $\mathrm{CaCl}_{2}(=137$ m.eq.).

of ammonia + urea nitrogen, on the other hand, the $C_{U r+N H_{2}}$ values were within or near the range of clearances obtained from the subject under ordinary dietary conditions. The creatinine clearances (Table II) indicated, like the ammonia t urea clearances, no significant decrease of renal function during the periods of high ammonia : urea ratios in the urine.

It therefore appears that, when acidosis increased ammonia excretion until it was important in comparison with urea excretion, the state of renal function was more accurately indicated by the urea clearance calculated on the assumption that the ammonia + urea of the urine represent the urea cleared from the blood. Calculation on the basis of excreted urea alone in this condition gave clearance values which were abnormally low for the subjects.
Comparison of clearances of urea and of urea + ammonia in normal subjects and nephritics, without induced acidosis. Table III gives the results obtained from a series of subjects on ordinary diets. The nephritic patients are divided into two groups, according to whether their clearances were above or below 20 per cent of average normal. It is evident that the ammonia : urea ratio in the urine tends to decrease as renal destruction becomes greater. The ammonia nitrogen averaged 5 per cent of the urea nitrogen in the normal subjects, 3.5 per cent in those nephritics who showed clearances above 20 per cent of normal, and only 1 per cent in the terminal cases, with clearances less than 10 per cent of normal. The highest ammonia: urea nitrogen ratio in the entire series was 1.09 in normal subject Number 2 . 
TABLE II

Comparison of clearances of urea, urea $+\mathrm{NH}_{2}$, hypobromite nitrogen, and creatinine, before and during induced acidosis. Subject G. Hypertension with normal clearance

\begin{tabular}{|c|c|c|c|c|c|c|c|c|c|c|c|c|c|}
\hline \multirow[b]{2}{*}{ Date } & \multirow[b]{2}{*}{$\begin{array}{l}\text { Protein } \\
\text { in diet }\end{array}$} & \multirow[b]{2}{*}{$\begin{array}{l}\text { Acidifying } \\
\text { salt taken }\end{array}$} & \multirow{2}{*}{$\begin{array}{c}\text { Urine } \\
\text { volume } \\
\text { corrected } \\
\text { for } \\
\text { body } \\
\text { size }\end{array}$} & \multicolumn{3}{|c|}{ Nitrogen per $100 \mathrm{cc}$. urine } & \multirow{2}{*}{$\begin{array}{c}\text { Urea } \\
\text { nitrogen } \\
\text { per } \\
\text { 100 cc. } \\
\text { blood by } \\
\text { urease } \\
\text { on whole } \\
\text { blood }\end{array}$} & \multicolumn{3}{|c|}{$\begin{array}{l}\text { Clearances, calculated } \\
\text { in percentages of mean } \\
\text { normal urea clearance }\end{array}$} & \multirow{2}{*}{$\begin{array}{l}\text { Plasma } \\
\text { creat- } \\
\text { inine } \\
\text { clear- } \\
\text { ance }\end{array}$} & \multirow{2}{*}{$\begin{array}{c}\text { Excretion } \\
\text { rate of } \\
\text { urea + NH3 } \\
\text { nitrogen. } \\
\text { Average } \\
\text { of } 2 \text { periods }\end{array}$} & \multirow{2}{*}{$\begin{array}{c}\text { Ve- } \\
\text { nous } \\
\text { plas- } \\
\text { ma } \\
\mathrm{CO}_{2}\end{array}$} \\
\hline & & & & $\left|\begin{array}{c}\text { NH, by } \\
\text { Van } \\
\text { Slyke- } \\
\text { Cullen } \\
\text { aeration }\end{array}\right|$ & $\begin{array}{c}\text { Urea } \\
\text { by } \\
\text { urease }\end{array}$ & $\begin{array}{c}\text { Hypo- } \\
\text { bromite } \\
\text { nitrogen }\end{array}$ & & $c_{U r}$ & $C_{U r+N H_{3}}$ & $C_{H y}$ & & & \\
\hline & $\begin{array}{l}\text { grams } \\
\text { per } \\
24 \text { hours }\end{array}$ & & $\begin{array}{c}c c . \\
\text { per } \\
\text { minute }\end{array}$ & $m g m$. & $m g m$. & mgm. & mgm. & $\begin{array}{l}\text { per } \\
\text { cent }\end{array}$ & $\begin{array}{l}\text { per } \\
\text { cent }\end{array}$ & $\begin{array}{l}\text { per } \\
\text { cent }\end{array}$ & $\underset{\text { cent* }}{\text { per }}$ & $\begin{array}{l}\text { grams } \\
\text { per } \\
24 \text { hours }\end{array}$ & $\begin{array}{c}m M . \\
\text { per } \\
\text { liter }\end{array}$ \\
\hline \multicolumn{14}{|c|}{ No acidosis } \\
\hline May 30 & Ad. lib. & None & $\begin{array}{l}1.21 \\
3.55\end{array}$ & $\begin{array}{r}22.1 \\
6.1\end{array}$ & $\begin{array}{l}892 \\
341\end{array}$ & $\begin{array}{l}885 \\
342\end{array}$ & 19.9 & $\begin{array}{l}91 \\
81\end{array}$ & $\begin{array}{l}94 \\
83\end{array}$ & $\begin{array}{l}91 \\
82\end{array}$ & & 16.9 & \\
\hline May 31 & Ad. lib. & None & $\begin{array}{l}4.09 \\
4.44\end{array}$ & $\begin{array}{l}8.4 \\
4.3\end{array}$ & $\begin{array}{l}269 \\
143\end{array}$ & $\begin{array}{l}253 \\
147\end{array}$ & 16.1 & $\begin{array}{l}91 \\
53\end{array}$ & $\begin{array}{l}94 \\
54\end{array}$ & $\begin{array}{l}86 \\
54\end{array}$ & $\begin{array}{l}88 \\
53\end{array}$ & 12.9 & \\
\hline June 1 & Ad. lib. & None & $\begin{array}{l}8.08 \\
2.22\end{array}$ & $\begin{array}{r}4.4 \\
12.4\end{array}$ & $\begin{array}{l}163 \\
421\end{array}$ & $\begin{array}{l}165 \\
419\end{array}$ & 15.2 & $\begin{array}{r}116 \\
82\end{array}$ & $\begin{array}{r}119 \\
85\end{array}$ & $\begin{array}{r}117 \\
82\end{array}$ & $\begin{array}{r}101 \\
79\end{array}$ & 11.7 & \\
\hline June 2 & Ad. lib. & None & $\begin{array}{l}8.88 \\
4.78\end{array}$ & $\begin{array}{l}3.5 \\
2.5\end{array}$ & $\begin{array}{r}77 \\
114\end{array}$ & $\begin{array}{r}78 \\
108\end{array}$ & 8.5 & $\begin{array}{r}107 \\
85\end{array}$ & $\begin{array}{r}112 \\
. \quad 87\end{array}$ & $\begin{array}{r}109 \\
81\end{array}$ & $\begin{array}{r}108 \\
72\end{array}$ & 9.3 & \\
\hline June 3 & 20 & $\mathrm{NH}_{4} \mathrm{Cl}$ & $\begin{array}{l}9.06 \\
4.44\end{array}$ & $\begin{array}{l}3.6 \\
4.1\end{array}$ & $\begin{array}{r}59 \\
115\end{array}$ & $\begin{array}{r}62 \\
120\end{array}$ & 7.9 & $\begin{array}{l}90 \\
86\end{array}$ & $\begin{array}{l}95 \\
89\end{array}$ & $\begin{array}{l}95 \\
90\end{array}$ & $\begin{array}{r}119 \\
95\end{array}$ & 8.0 & \\
\hline \multicolumn{14}{|c|}{ Acidosis } \\
\hline June 5 & 20 & $\mathrm{NH}_{4} \mathrm{Cl}$ & $\begin{array}{l}4.80 \\
3.38\end{array}$ & $\begin{array}{l}20.4 \\
28.6\end{array}$ & $\begin{array}{l}109 \\
151\end{array}$ & $\begin{array}{l}126 \\
175\end{array}$ & 9.5 & $\begin{array}{l}73 \\
71\end{array}$ & $\begin{array}{l}87 \\
85\end{array}$ & $\begin{array}{l}85 \\
83\end{array}$ & $\begin{array}{r}106 \\
75\end{array}$ & 8.9 & 20.4 \\
\hline June 7 & 20 & $\mathrm{NH}_{4} \mathrm{Cl}$ & $\begin{array}{l}4.63 \\
4.09\end{array}$ & $\begin{array}{l}29.1 \\
30.1\end{array}$ & $\begin{array}{l}99 \\
92\end{array}$ & $\begin{array}{l}122 \\
116\end{array}$ & 11.2 & $\begin{array}{l}55 \\
44\end{array}$ & $\begin{array}{l}71 \\
60\end{array}$ & $\begin{array}{l}68 \\
57\end{array}$ & $\begin{array}{l}93 \\
57\end{array}$ & 7.9 & 17.8 \\
\hline June 10 & 20 & $\mathrm{NH}_{4} \mathrm{Cl}$ & $\begin{array}{l}1.16 \\
3.20\end{array}$ & $\begin{array}{r}175.9 \\
64.2\end{array}$ & $\begin{array}{l}373 \\
163\end{array}$ & $\begin{array}{l}522 \\
212\end{array}$ & 11.1 & $\begin{array}{l}67 \\
63\end{array}$ & $\begin{array}{l}99 \\
88\end{array}$ & $\begin{array}{l}94 \\
83\end{array}$ & $\begin{array}{r}105 \\
74\end{array}$ & 9.8 & 16.4 \\
\hline June 11 & 20 & $\mathrm{NH}_{4} \mathrm{Cl}$ & $\begin{array}{l}1.96 \\
1.69\end{array}$ & $\begin{array}{l}116.7 \\
130.3\end{array}$ & $\begin{array}{l}237 \\
286\end{array}$ & $\begin{array}{l}340 \\
403\end{array}$ & 12.3 & $\begin{array}{l}61 \\
56\end{array}$ & $\begin{array}{l}74 \\
81\end{array}$ & $\begin{array}{l}71 \\
79\end{array}$ & $\begin{array}{l}81 \\
66\end{array}$ & 10.1 & \\
\hline \multicolumn{8}{|c|}{ Mean and standard deviation of clearances May 30 to June 3} & $\begin{array}{r}88 \\
\pm 15\end{array}$ & $\begin{array}{r}91 \\
\pm 16\end{array}$ & $\begin{array}{r}89 \\
\pm 15\end{array}$ & $\begin{array}{r}89 \\
\pm 21\end{array}$ & & \\
\hline \multicolumn{8}{|c|}{ Mean and standard deviation of clearances June 5 to June 11} & $\begin{array}{r}61 \\
\pm 10\end{array}$ & $\begin{array}{r}81 \\
\pm 12\end{array}$ & $\begin{array}{r}78 \\
\pm 11\end{array}$ & $\begin{array}{r}82 \\
\pm 18\end{array}$ & & \\
\hline
\end{tabular}

* For plasma creatinine clearance $148 \mathrm{cc}$. per minute is taken as 100 per cent of mean normal.

If, in calculating the $\mathrm{CUr}_{r}+\mathrm{NH}_{3}$ values, the factors 1.33 and 1.85 in Formulae 1 and 2 were reduced by 5 per cent, to 1.26 and 1.76 respectively, the $\mathrm{CU}_{\mathrm{r}}+\mathrm{NH}_{3}$ values, calculated from excretion of urea + ammonia, would in no case in Table III deviate by more than 4 per cent from the true $C U_{r}$ values, calculated from the excretion of urea alone.

Comparison of the urea clearance with the clearance of nitrogen determinable by hypo- bromite, in nephritic patients with varying degrees of renal impairment. Table IV shows that, in the 54 urines analyzed, the nitrogen determinable by hypobromite in the urine averaged ex. actly the same as the urea nitrogen determined with urease. In all but 2 urines the two methods of analysis gave within 5 per cent the same values. In one case the hypobromite method gave 6 per cent more, and in one 12 per cent more than the urea nitrogen. 
These are the results to be expected. As shown in the original description of the manometric hypobromite method (Van Slyke (1929)),

TABLE III

Comparison of clearances of urea and of urea + ammonia in normal subjects and in nephritics with varying degrees of renal impairment

Urea in blood and urine by gasometric urease- $\mathrm{CO}_{2}$ method (Van Slyke (1927)). Ammonia in urine by aeration and titration (Van Slyke and Cullen (1914)).

\begin{tabular}{|c|c|c|c|c|}
\hline Number & Initials & $\begin{array}{c}\text { Urea } \\
\text { clearance } \\
C_{U r}\end{array}$ & $\begin{array}{l}\text { Urea }+\mathrm{NH}_{3} \\
\text { clearance } \\
C_{U r}+N H_{3}\end{array}$ & $\begin{array}{l}\text { Ratio of } \\
C_{U r}+N H_{3} \\
\text { to } C_{U r}\end{array}$ \\
\hline & & $\begin{array}{l}\text { per cent of } \\
\text { normal } C U r\end{array}$ & $\begin{array}{c}\text { per cent of } \\
\text { normal } C U r\end{array}$ & \\
\hline \multicolumn{5}{|c|}{ Normal subjects } \\
\hline 1 & E.K. & $\begin{array}{l}130 \\
164\end{array}$ & $\begin{array}{l}137 \\
168\end{array}$ & $\begin{array}{l}1.06 \\
1.03\end{array}$ \\
\hline 2 & B. & $\begin{array}{r}65 \\
100 \\
101\end{array}$ & $\begin{array}{r}72 \\
102 \\
110\end{array}$ & $\begin{array}{l}1.06 \\
1.02 \\
1.09\end{array}$ \\
\hline \multirow[t]{2}{*}{3} & J.M. & $\begin{array}{l}118 \\
117 \\
111\end{array}$ & $\begin{array}{l}125 \\
124 \\
115\end{array}$ & $\begin{array}{l}1.06 \\
1.06 \\
1.03\end{array}$ \\
\hline & \multicolumn{3}{|r|}{ Average } & 1.05 \\
\hline
\end{tabular}

Nephritic patients with clearances above 20 per cent

\begin{tabular}{|c|c|c|c|c|}
\hline 1 & A.Ch. & $\begin{array}{l}72 \\
70 \\
83\end{array}$ & $\begin{array}{l}74 \\
72 \\
85\end{array}$ & $\begin{array}{l}1.03 \\
1.02 \\
1.03\end{array}$ \\
\hline 2 & A.Co. & $\begin{array}{l}53.6 \\
55.4 \\
37.1 \dagger \\
32.6 \dagger \\
44.6 \ddagger \\
23.3 \ddagger\end{array}$ & $\begin{array}{l}55.0 \\
56.0 \\
38.0 \\
33.1 \\
47.4 \\
24.8\end{array}$ & $\begin{array}{l}1.03 \\
1.01 \\
1.02 \\
1.02 \\
1.06 \\
1.06\end{array}$ \\
\hline 3 & McG. & $\begin{array}{l}75.9 \\
48.4 \\
31.8 \\
32.6 \\
32.0 \\
28.4\end{array}$ & $\begin{array}{l}77.1 \\
51.5 \\
34.4 \\
35.1 \\
34.0 \\
29.4\end{array}$ & $\begin{array}{l}1.01 \\
1.06 \\
1.08 \\
1.08 \\
1.06 \\
1.04\end{array}$ \\
\hline 4 & Ra. & $\begin{array}{l}28.4 \\
38.9\end{array}$ & $\begin{array}{l}28.9 \\
39.9\end{array}$ & $\begin{array}{l}1.02 \\
1.02\end{array}$ \\
\hline 5 & Cal. & $\begin{array}{l}29.1 \\
24.5\end{array}$ & $\begin{array}{l}29.4 \\
24.8\end{array}$ & $\begin{array}{l}1.01 \\
1.01\end{array}$ \\
\hline 6 & Or. & $\begin{array}{l}18.7 \\
22.0\end{array}$ & $\begin{array}{l}19.5 \\
22.6\end{array}$ & $\begin{array}{l}1.04 \\
1.03\end{array}$ \\
\hline \multicolumn{4}{|r|}{ Average } & 1.035 \\
\hline
\end{tabular}

$\dagger$ Low protein diet, 20 grams per day.

$\ddagger$ High protein diet, 130 grams per day.
TABLE III-continued

\begin{tabular}{c|c|c|c|c}
\hline Number & Initials & $\begin{array}{c}\text { Urea } \\
\text { clearance } \\
C_{U r}\end{array}$ & $\begin{array}{c}\text { Urea }+N_{3} \\
\text { clearance } \\
C_{U r}+N H_{3}\end{array}$ & $\begin{array}{c}\text { Ratio of } \\
C U r+N H_{3} \\
\text { to } C_{U r}\end{array}$ \\
\hline & $\begin{array}{c}\text { per cent of } \\
\text { normal } C_{U r}\end{array}$ & $\begin{array}{c}\text { per cent of } \\
\text { normal } C_{U r}\end{array}$ & \\
\hline
\end{tabular}

Nephritic patients with clearances below 20 per cent

\begin{tabular}{|c|c|c|c|c|}
\hline 1 & Fl. & $\begin{array}{l}9.00 \\
8.30 \\
7.70 \\
8.20 \\
9.20 \\
9.10\end{array}$ & $\begin{array}{l}9.20 \\
8.30 \\
7.80 \\
8.40 \\
9.40 \\
9.20\end{array}$ & $\begin{array}{l}1.02 \\
1.00 \\
1.01 \\
1.02 \\
1.02 \\
1.01\end{array}$ \\
\hline 2 & Car. & $\begin{array}{l}8.65 \\
8.65 \\
9.65\end{array}$ & $\begin{array}{l}8.78 \\
8.78 \\
9.78\end{array}$ & $\begin{array}{l}1.01 \\
1.01 \\
1.01\end{array}$ \\
\hline 3 & Jo. & $\begin{array}{l}7.50 \\
7.20\end{array}$ & $\begin{array}{l}7.67 \\
7.39\end{array}$ & $\begin{array}{l}1.02 \\
1.03\end{array}$ \\
\hline 4 & Wein. & $\begin{array}{l}5.79 \\
5.79\end{array}$ & $\begin{array}{l}5.88 \\
5.91\end{array}$ & $\begin{array}{l}1.02 \\
1.02\end{array}$ \\
\hline \multirow[t]{2}{*}{5} & Cap. & $\begin{array}{l}5.30 \\
6.88 \\
5.20 \\
3.69 \\
3.74\end{array}$ & $\begin{array}{l}5.41 \\
6.92 \\
5.24 \\
3.70 \\
3.75\end{array}$ & $\begin{array}{l}1.02 \\
1.01 \\
1.01 \\
1.00 \\
1.00\end{array}$ \\
\hline & & & Average & 1.01 \\
\hline
\end{tabular}

Comparison of clearances of hypobromite nitrogen and of urea nitrogen in nephritic patients with varying degrees of renal impairment

\begin{tabular}{|c|c|c|c|}
\hline Number & $\begin{array}{l}\text { Ratio of } \\
\text { urea nitrogen to } \\
\text { hypobromite } \\
\text { nitrogen } \\
\text { in urine* }\end{array}$ & $\begin{array}{c}\text { Urea } \\
\text { clearance } \\
C_{U r}\end{array}$ & $\begin{array}{c}\text { Hypobromite } \\
\text { nitrogen } \\
\text { clearance } \\
C_{H y}\end{array}$ \\
\hline 1 & $\begin{array}{l}1.01 \\
1.01\end{array}$ & $\begin{array}{c}\text { per cent of } \\
\text { normal } C U \text { r } \\
151 \\
129\end{array}$ & $\begin{array}{c}\text { per cent of } \\
\text { normal } C U \text { r } \\
153 \\
130\end{array}$ \\
\hline 2 & $\begin{array}{l}1.01 \\
1.00\end{array}$ & $\begin{array}{l}188 \\
110\end{array}$ & $\begin{array}{l}190 \\
110\end{array}$ \\
\hline 3 & 0.96 & 106 & 103 \\
\hline 4 & $\begin{array}{l}1.04 \\
1.04\end{array}$ & $\begin{array}{l}112 \\
112\end{array}$ & $\begin{array}{l}117 \\
117\end{array}$ \\
\hline 5 & $\begin{array}{l}1.02 \\
0.97\end{array}$ & $\begin{array}{r}90 \\
107\end{array}$ & $\begin{array}{r}92 \\
104\end{array}$ \\
\hline 6 & $\begin{array}{l}0.94 \\
0.97\end{array}$ & $\begin{array}{l}93 \\
84\end{array}$ & $\begin{array}{l}88 \\
82\end{array}$ \\
\hline 7 & $\begin{array}{l}1.00 \\
0.99\end{array}$ & $\begin{array}{l}83 \\
65\end{array}$ & $\begin{array}{l}83 \\
64\end{array}$ \\
\hline
\end{tabular}

* This urine concentration ratio is also the clearance ratio, $C_{U r}: C_{H y}$. 
TABLE IV-continued

\begin{tabular}{|c|c|c|c|}
\hline Number & $\begin{array}{l}\text { Ratio of } \\
\text { urea nitrogen to } \\
\text { hypobromite } \\
\text { nitrogen } \\
\text { in urine* }\end{array}$ & $\begin{array}{c}\text { Urea } \\
\text { clearance } \\
C_{U_{r}}\end{array}$ & $\begin{array}{c}\text { Hypobromite } \\
\text { nitrogen } \\
\text { clearance } \\
C_{H y}\end{array}$ \\
\hline & & $\begin{array}{l}\text { per cent of } \\
\text { normal } C_{U r}\end{array}$ & $\begin{array}{l}\text { per cent of } \\
\text { normal } C_{U r}\end{array}$ \\
\hline 8 & $\begin{array}{l}0.97 \\
0.97\end{array}$ & $\begin{array}{l}89 \\
71\end{array}$ & $\begin{array}{l}85 \\
69\end{array}$ \\
\hline 9 & $\begin{array}{l}0.96 \\
1.02\end{array}$ & $\begin{array}{l}87 \\
67\end{array}$ & $\begin{array}{l}84 \\
68\end{array}$ \\
\hline 10 & $\begin{array}{l}1.01 \\
1.00\end{array}$ & $\begin{array}{l}95 \\
75\end{array}$ & $\begin{array}{l}96 \\
75\end{array}$ \\
\hline 11 & $\begin{array}{l}0.98 \\
0.94\end{array}$ & $\begin{array}{l}72 \\
73\end{array}$ & $\begin{array}{l}71 \\
69\end{array}$ \\
\hline 12 & $\begin{array}{l}1.01 \\
1.00\end{array}$ & $\begin{array}{l}69 \\
69\end{array}$ & $\begin{array}{l}71 \\
69\end{array}$ \\
\hline 13 & $\begin{array}{l}1.05 \\
1.12\end{array}$ & $\begin{array}{l}55 \\
56\end{array}$ & $\begin{array}{l}58 \\
63\end{array}$ \\
\hline 14 & $\begin{array}{l}1.01 \\
1.02\end{array}$ & $\begin{array}{l}47 \\
57\end{array}$ & $\begin{array}{l}47 \\
58\end{array}$ \\
\hline 15 & $\begin{array}{l}0.98 \\
0.99\end{array}$ & $\begin{array}{l}48 \\
41\end{array}$ & $\begin{array}{l}47 \\
41\end{array}$ \\
\hline 16 & $\begin{array}{l}0.98 \\
1.01\end{array}$ & $\begin{array}{l}44 \\
40\end{array}$ & $\begin{array}{l}43 \\
40\end{array}$ \\
\hline 17 & $\begin{array}{l}0.97 \\
0.96\end{array}$ & $\begin{array}{l}30 \\
33\end{array}$ & $\begin{array}{l}30 \\
30\end{array}$ \\
\hline 18 & $\begin{array}{l}1.05 \\
1.02\end{array}$ & $\begin{array}{l}26 \\
37\end{array}$ & $\begin{array}{l}27 \\
38\end{array}$ \\
\hline 19 & $\begin{array}{l}0.99 \\
1.00\end{array}$ & $\begin{array}{l}27 \\
33\end{array}$ & $\begin{array}{l}27 \\
33\end{array}$ \\
\hline 20 & $\begin{array}{l}1.00 \\
1.03\end{array}$ & $\begin{array}{l}23.1 \\
22.4\end{array}$ & $\begin{array}{l}23.0 \\
23.1\end{array}$ \\
\hline 21 & $\begin{array}{l}0.97 \\
0.97\end{array}$ & $\begin{array}{l}15.8 \\
18.6\end{array}$ & $\begin{array}{l}15.4 \\
18.1\end{array}$ \\
\hline 22 & $\begin{array}{l}1.01 \\
0.98\end{array}$ & $\begin{array}{l}17.2 \\
16.0\end{array}$ & $\begin{array}{l}17.3 \\
15.8\end{array}$ \\
\hline 23 & $\begin{array}{l}0.97 \\
0.98\end{array}$ & $\begin{array}{l}15.3 \\
15.9\end{array}$ & $\begin{array}{l}14.8 \\
15.6\end{array}$ \\
\hline 24 & $\begin{array}{l}1.01 \\
1.03\end{array}$ & $\begin{array}{l}13.3 \\
14.1\end{array}$ & $\begin{array}{l}13.4 \\
14.5\end{array}$ \\
\hline 25 & $\begin{array}{l}1.02 \\
1.02\end{array}$ & $\begin{array}{l}12.8 \\
14.2\end{array}$ & $\begin{array}{l}13.0 \\
14.4\end{array}$ \\
\hline 26 & $\begin{array}{l}1.00 \\
1.01\end{array}$ & $\begin{array}{l}14.6 \\
10.3\end{array}$ & $\begin{array}{l}14.6 \\
10.4\end{array}$ \\
\hline 27 & $\begin{array}{l}0.95 \\
1.06\end{array}$ & $\begin{array}{r}9.4 \\
10.2\end{array}$ & $\begin{array}{r}8.9 \\
10.8\end{array}$ \\
\hline 28 & 0.98 & 2.2 & 2.2 \\
\hline Average ratio & 1.00 & & \\
\hline
\end{tabular}

the nitrogen yielded by it from urea, under the conditions prescribed for urine analysis, is 95 per cent of theoretical. With ammonia the yield is almost the same, 96 per cent (Van Slyke (1927)). As the ammonia nitrogen in the urine ordinarily averages about 5 per cent of the urea nitrogen (see for example Table II), one could expect that, when the hypobromite reagent is applied with the urinary ammonia present, the $\mathrm{N}_{2}$ gas yielded by the ammonia would, on the average, make up the 5 per cent deficit in the $\mathrm{N}_{2}$ yielded by the urea.

Hypobromite clearances less than 95 per cent of the urea clearance cannot occur, except as the result of analytical error, because the hypobromite reagent gives 95 per cent of theoretical $\mathrm{N}_{2}$ from pure urea. (It also evolves some $\mathrm{N}_{2}$ from other urinary substances, such as uric acid and creatine, but their effect on the result in human urine appears to be ordinarily slight.)

Hypobromite nitrogen clearances above 105 per cent of the urea clearance will occur when the ammonia nitrogen exceeds 10 per cent of the urea, e.g., Number 13, Table IV. In such cases the hypobromite nitrogen clearance will approximate (95 per cent or a little more) the clearance of urea + ammonia nitrogen, which has been shown above to be a more consistent measure of renal function than the clearance of urea alone.

It appears that the comparative significance of the urea clearance and the hypobromite nitrogen clearance may be summarized as follows.

1. The average normal values yielded by both are identical, hence the same standard normal values, heretofore used for the urea clearance, apply without change to the hypobromite nitrogen clearance. In calculating percentages of normal values for the hypobromite nitrogen clearance, the factors 1.33 and 1.85 , of Formulae 1 and 2, can therefore be used without change, also the nomograms for graphic calculation of the clearance. (Möller, McIntosh and Van Slyke $(1928, a)$; Peters and Van Slyke (1932), pp. 566 and 567.)

2 . The hypobromite nitrogen clearance will always be 95 per cent or more of the urea clearance.

3 . When, by reason of an unusually high ammonia : urea ratio in urine, the hypobromite nitrogen clearance significantly exceeds the urea clearance, the hypobromite nitrogen clearance may be 
taken as the more accurate measure of renal function, since it approximates the urea + ammonia clearance, which has been found to be more consistent than the simple urea clearance.

From these conclusions it appears that the hypobromite nitrogen clearance can be taken not only as a technically convenient, but also as an accurate measure of renal function, practically equalling for this purpose the true urea clearance when the ammonia: urea ratio in the urine is within ordinary limits, and excelling the urea clearance when the relative ammonia excretion is exceptionally great.

\section{Present urea clearance procedure in this Clinic}

Experience accumulated since the details for determining the urea clearance were first published (Möller, McIntosh and Van Slyke (1928, a)) has somewhat more completely indicated the conditions desirable for the procedure, and we will briefly outline the manner in which it is now carried out.

Preparation of the subject. Except in fairly advanced cases of nephritis, it ordinarily makes no significant difference whether the subject lies down during the test or walks about. Van Slyke, Alving and Rose (1932) found that it made no difference in any subject examined by them who showed more than 50 per cent of normal clearance, but that 3 out of 12 nephritics with less than 50 per cent normal function showed lower clearances if they were up and about. It is therefore desirable that nephritics, who have advanced to less than 50 per cent of normal clearance, rest in a reclining posture during the test, but in other subjects it is not essential.

It is desirable to promote a fairly free flow of urine during the test, in order to diminish the relative error caused by retention of urine in the bladder at the voidings. For this purpose we routinely give two glasses of water, one at the beginning of the test, and another an hour later, after the first specimen of urine has been collected. This is the only special preparation of the subject which we routinely follow. It may be omitted when for any reason it is desirable to do so.

The gain in constancy of results caused by increasing the urine volume is indicated by the following results.
Möller, McIntosh and Van Slyke (1928, a) found that the mean probable deviation, in a given subject, from the average clearance of that subject, was \pm 9.2 per cent when the urine volume was under $2 \mathrm{cc}$. per minute, and \pm 7.0 per cent when the volume exceeded 2 cc. Addis (1922) obtained routinely a great diuresis by giving one liter of water, with 15 grams of urea, 3 hours before the test, and two glasses (about $400 \mathrm{cc}$.) each hour thereafter until the test was completed. Under these conditions the mean probable deviation of the clearance in an individual was reduced to \pm 3.7 per cent.

Some of the earlier studies of urea excretion indicated that coffee affected the results (Addis and Drury (1923)), and that clearances taken in the morning might be more consistent than those taken at other times of the day. Page (1933) has found, however, that the amount of caffeine in an ordinary cup of coffee has no measurable effect on the clearance. And general experience in this hospital has shown that the time of day also has no significant effect. The clearance may therefore be taken whenever it is most convenient, and it is not necessary to forbid the taking of a single cup of coffee or of ordinary amounts of food before the test.

Addis and Watanabe (1916) and Addis (1922) gave 15 grams of urea routinely, except in cases with blood urea already high, with the idea that the increased load of urea would put the kidney under a strain and reveal functional deficits that might otherwise be missed. Fowweather (1934) also recommends urea administration on the basis of his experience, but for exactly the opposite reason; giving the urea appeared to prevent an occasional erroneously low clearance which was encountered when urea was not given. Cope (1934), apparently influenced by Fowweather's results, advises the giving of urea. Möller, McIntosh and Van Slyke (1928, $a$ and $b$ ), however, found that the urea administration had no influence, either in raising or lowering the clearance, either in normal or in nephritic subjects. Later, Van Slyke, Alving and Rose (1932) again tested the question carefully, and likewise concluded that urea administration was without effect on the clearance values. It is the writers' belief that the administration of urea increases the accuracy of the clearance determinations only when, by increasing the blood urea content (Fowweather found an average increase of $16 \mathrm{mgm}$. urea nitrogen per $100 \mathrm{cc}$. blood after giving 15 grams of urea), it augments the accuracy of the blood urea determinations. When a technique for blood urea is used which gives satisfactorily accurate results with the concentrations of blood urea naturally present, there appears to be nothing gained by urea administration. It complicates the procedure, and the methods for rapid blood analysis now available are sufficiently accurate to make the addition unnecessary. One of the advantages of the urea clearance as a renal test is that it is not necessary to complicate it by administration of anything.

Collecting the urine. It is desirable to collect two specimens of urine, each over a measured 
period, for which about an hour is a satisfactory time. A single specimen of blood for analysis drawn near the middle of the two-hour interval serves for comparison with both urine specimens. In consequence, two clearances are measured with only one drawing of blood.

To start the first period, the subject, after drinking his first glass of water, empties his bladder completely, without saving the specimen, and a stopwatch is started. At the end of approximately an hour the subject again voids completely and the specimen is saved (Period 1).

At the moment when the subject finishes voiding, the time on the stopwatch is recorded, as the first period, and the watch is immediately restarted for the second period. The subject then drinks his second glass of water.

At approximately the end of the second hour he again voids completely, the time of this period is accurately recorded, and the specimen is saved (Period 2).

It is not necessary that the time of each period shall be exactly an hour; it may without harm be longer, up to several hours; or it may be shorter than an hour, provided enough urine (preferably over $50 \mathrm{cc}$.) is voided to prevent undue error from the volume retained. The essential things are, that the time be exactly recorded, that the approximately complete urine content of the bladder be obtained, and that the blood urea determined represent approximately the average for the period.

In some patients it is impossible to obtain urination at will. In such cases one may continue the collection period for several hours until spontaneous urination occurs, provided the time is accurately recorded. Especially in young children, it is frequently necessary to wait for spontaneous urination. For these the automatic recording device described in the accompanying paper by Farr (1935) is a great advantage.

Urine measurement. The urine from each period is measured within 1 per cent. Accurate graduated cylinders of 25,50,100 and $200 \mathrm{cc}$. are kept at hand, and the smallest is used which will contain the specimen.

Collecting blood. At about the end of the first hour of the usual 2-hour test, a sample of blood is drawn for analysis. Ordinarily, when no urea has been administered, the change of blood urea content is slow, so that a matter of 10 or $15 \mathrm{~min}$ utes time, or even more, sooner or later, is not important. We usually draw 2 cc. or more of blood from a vein into a syringe, thereby obtaining sufficient for duplicates by the usual gasometric methods. When, however, economy of blood is desirable, $0.2 \mathrm{cc}$. is taken from a finger or ear, or, for small children from the heel as described by Drucker and Cullen (1925), and the urea is determined by micro-analysis.

Urine analysis. The rapid hypobromite urea method with the manometric apparatus is used (Van Slyke (1929), Peters and Van Slyke (1932), p. 379). The treatment with permutit to remove ammonia, included in the original description of the method, is omitted for reasons developed in this paper. The urine is merely diluted 10 or 20 -fold, a sample is measured into the manometric chamber, hypobromite is added, the chamber is shaken $1.5,2$ or 3 minutes at $25^{\circ}, 20^{\circ}$, or $15^{\circ}$, respectively, and the analysis is finished by taking the reading. The improved hypobromite reagent of Van Slyke and Kugel (1933) is used.

Analysis of blood. The manometric hypobromite method is also routinely used for blood urea. It is applied, as described by Van Slyke and Kugel (1933), to the blood filtrate obtained by deproteinization with zinc hydroxide according to the procedure of Somogyi (1930). The correction of 1.2 mgm., recommended by Van Slyke and Kugel for $\mathrm{N}_{2}$ evolved from non-urea substances in the Somogyi blood filtrate, is applied. As found by Van Slyke and Kugel, the maximum error by this method is $0.5 \mathrm{mgm}$. of urea nitrogen per $100 \mathrm{cc}$. of blood. The method is simpler. with regard to reagents and procedure than the urease methods, and is as accurate as the urease procedure recommended for routine analyses ("Procedure A, for determinations in a series of blood filtrates," p. 703, Van Slyke (1927)).

For micro-analyses of blood drawn from skin puncture, the micro-gasometric urease method (Van Slyke (1927)) has until lately been employed. However, a micro-hypobromite modification has been developed by Farr, and is described in his accompanying paper (1935).

Calculation. When the urinary hypobromite nitrogen is determined as above described, the clearance is calculated by Formulae 1 or 2, with 
the urine nitrogen determined by the hypobromite representing $U$ in the formulae. The same nomograms (Möller, McIntosh and Van Slyke (1928, a) ; Peters and Van Slyke (1932), pp. 566 and 567) used for facilitating calculation of the clearance from exact urinary urea values are used also with the hypobromite nitrogen values.

If laboratory conveniences should make it easier to determine urea + ammonia nitrogen (e.g., by the urease method, with determination of the preformed ammonia together with the ammonia formed from urea) one would use the formulae with the constants 1.26 and 1.76 substituted for 1.33 and 1.85 .

Clearance by direct colorimetric comparison of blood and urine urea. (Van Slyke and Cope (1932).) This method is convenient where laboratory facilities make colorimetry more practicable than manometric analysis. Because the reverse is the case in this laboratory, the colorimetric procedure has not been used routinely by the writers. However, it has given satisfactory routine service in the clinic of one of our colleagues (Prof. L. H. Newburgh).

In the light of results in the present paper, the colorimetric clearance may be simplified by omitting the preliminary treatment of the urine with permutit to remove ammonia, and by making the urine dilutions to 1.05 times the volumes indicated by the graph of Van Slyke and Cope.

\section{SUM MARY}

Experiments with human subjects show that when the proportion of urea in the urea + ammonia mixture of the urine is markedly decreased by induced acidosis and low protein diet, the urea clearances calculated from the excretion rate of urea alone suffer a parallel reduction. If, however, values for excretion of urea + ammonia are substituted for urea, the clearances calculated remain at the usual levels.

The theoretical significance of the results is to favor the hypotheses, that the ammonia excreted in the urine of man is formed in the kidneys chiefly from urea removed from the blood, and that the work of the kidneys in excreting urea from the blood is more accurately indicated by the combined excretion of urea and ammonia than by the excretion of urea alone.
The practical deduction is that in determinations of the urea clearance as a measure of renal function, results are somewhat more consistent if in the clearance formula, $\frac{U V}{B}$ or $\frac{U \sqrt{V}}{B}$, one uses for $U$ the urinary concentration of urea + ammonia nitrogen, instead of only urea nitrogen. When the urinary urea is determined by methods measuring the $\mathrm{NH}_{3}$ formed by urea hydrolysis, or the $\mathrm{N}_{2}$ yielded by the action of hypobromite, the determination of combined urea + ammonia nitrogen is also simpler than determining the urea nitrogen separately.

The routine procedure developed for clinical determination of the urea clearance is described.

\section{BIBLIOGRAPHY}

Addis, T., Renal function and the amount of functioning tissue. Arch. Int. Med., 1922, 30, 378.

Addis, T., and Drury, D. R., The rate of urea excretion. VII. The effect of various factors other than blood urea concentration on the rate of urea excretion. J. Biol. Chem., 1923, 55, 629.

Addis, T., and Watanabe, C. K., A method for the measurement of the urea-excreting function of the kidneys. J. Biol. Chem., 1916, 27, 251.

Bell, L. G., Gilmour, C. R., and Cameron, A. T., Clinical studies with the urea clearance test. Canad. M. A. J., 1934, 30, 246.

Cope, C. L., The rational assessment of renal damage. Lancet, 1934, 2, 799.

Drucker, P., and Cullen, G. E., A simple method for obtaining cutaneous (capillary) blood from infants and adults for colorimetric $\mathrm{pH}$ determinations. J. Biol. Chem., 1925, 64, 221.

Farr, L., A micro method for blood urea and an automatic urine collector for urea clearance in infants. J. Clin. Invest., 1935, 14, 911.

Fowweather, F. S., Blood urea clearances before and after giving urea. Quart. J. Med., 1934, 27, 63.

Krebs, H. A., Untersuchungungen ueber den Stoffwechsel der Aminosaueren im Tierkoerper. Ztschr. f. physiol. Chem., 1933, 217, 191; 218, 158.

McIntosh, J. F., Möller, E., and Van Slyke, D. D., Studies of urea excretion. III. The influence of body size on urea output. J. Clin. Invest., 1928, 6, 467.

Möller, E., McIntosh, J. F., and Van Slyke, D. D., Studies of urea excretion. II. Relationship between urine volume and the rate of urea excretion by adults. J. Clin. Invest., 1928a, 6, 427.

Möller, E., McIntosh, J. F., and Van Slyke, D. D., Studies of urea excretion. IV. Relationship between urine volume and rate of urea excretion in patients with Bright's disease. J. Clin. Invest., 1928b, 6, 485.

Nash, T. P., and Benedict, S. R., The ammonia content 
of the blood and its bearing on the mechanism of acid neutralization in the animal organism. J. Biol. Chem., 1921, 48, 463.

Page, I. H., Action of certain diuretics on the function of the kidney as measured by the urea clearance test. J. Clin. Invest., 1933, 12, 737.

Peters, J. P., and Van Slyke, D. D., Quantitative Clinical Chemistry. I. Interpretations, 1931. II. Methods, 1932, Williams and Wilkins Co., Baltimore.

Somogyi, M., A method for the preparation of blood filtrates for the determination of sugar. J. Biol. Chem., 1930, 86, 655.

Van Slyke, D. D., Determination of urea by gasometric measurement of the carbon dioxide formed by the action of urease. J. Biol. Chem., 1927, 73, 695. (Also Peters and Van Slyke, II, p. 360.)

Van Slyke, D. D., The manometric determination of urea in blood and urine by the hypobromite reaction. J. Biol. Chem., 1929, 83, 449. (Also Peters and Van Slyke, II, 379.)

Van Slyke, D. D., Alving, A., and Rose, W. C., Studies of urea excretion. VII. The effects of posture and exercise on urea excretion. J. Clin. Invest., 1932, 11, 1053.

Van Slyke, D. D., and Cope, C. L., Simplified colorimetric determination of blood urea clearance. Proc. Soc. Exper. Biol. and Med., 1932, 29, 1169. (Also Peters and Van Slyke, II, p. 935.)

Van Slyke, D. D., and Cullen, G. E., A permanent preparation of urease and its use in the determination of urea. J. Biol. Chem., 1914, 19, 211 ; 1916, 24, 117.

Van Slyke, D. D., and Kugel, V. H., Improvements in manometric micro-Kjeldahl and blood urea methods. J. Biol. Chem., 1933, 102, 489. 